$$
\begin{aligned}
& \text { خوارزمية فتح المسار الأقصر والأقل اكتظاظاً أولاً في الثبكات الحاسوبية } \\
& \text { زينة ناطق عبات القادر }
\end{aligned}
$$

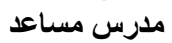

$$
\begin{aligned}
& \text { كلية التربية/قسم علوم الحاسوب }
\end{aligned}
$$

يعد بروتوكول فتح المسار الأقصر أولاً (Open Shortest Path First-OSPF) أحد بروتة فئوكولات

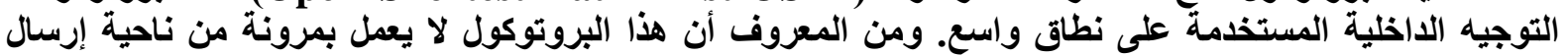

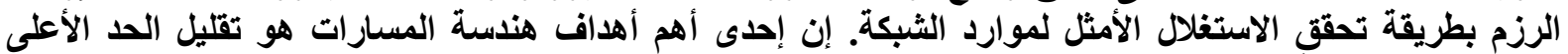

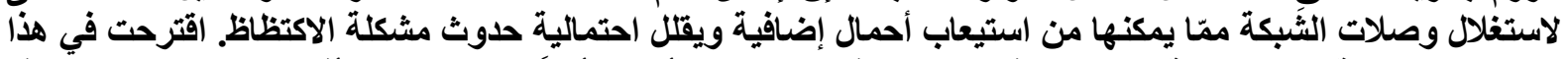

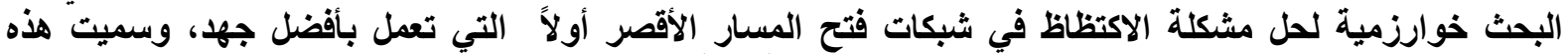

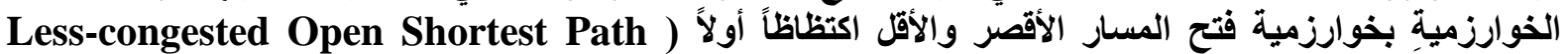

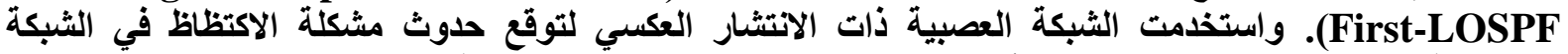

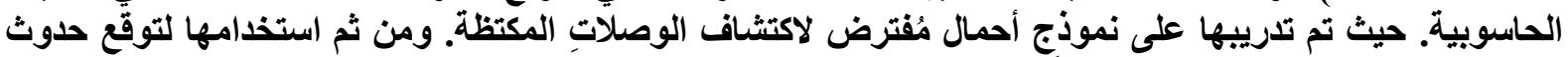

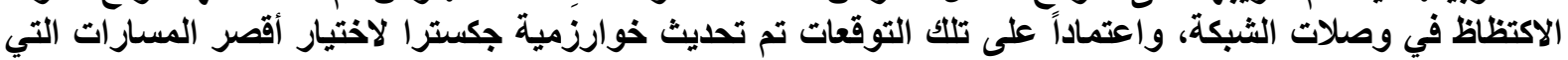

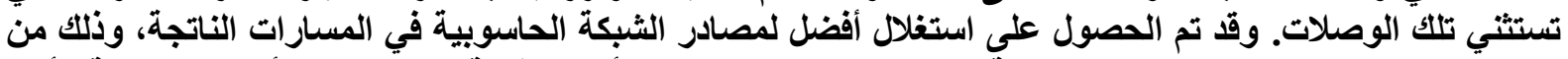

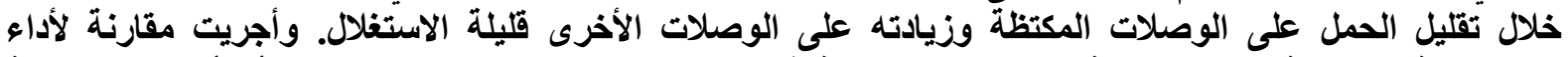

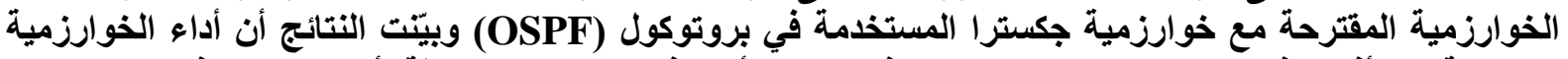

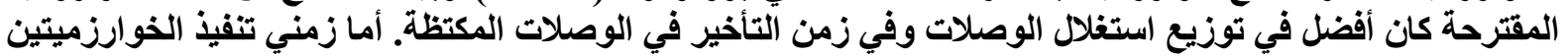

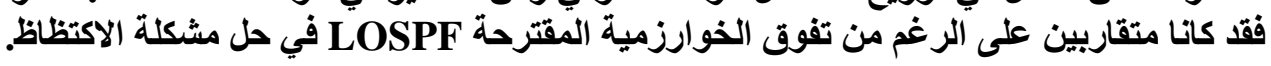

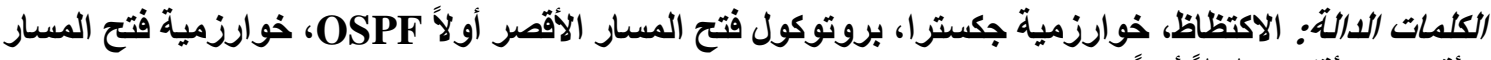

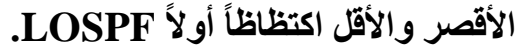

\title{
Less Congested Open Shortest Path First Algorithm for Computer Networks
}

\section{Dr. Manar Younis Kashmola} Ass. Prof.

College of Computer Sciences and Mathematics

\author{
Zena Natiq Abdul-Kader \\ Ass. Lecturer \\ College of Education
}

\section{Abstract}

Open Shortest Path First (OSPF) is one of the most widely used intra-domain routing protocol. It is well known that OSPF protocol does not provide flexibility in terms of packet forwarding to achieve any network optimization objective. The common objective of network traffic engineering is to minimize the maximal link utilization in a network in order to accommodate more traffic and reduce the chance of congestion In this paper an algorithm, to solve congestion problem in an OSPF based best effort network, is proposed. This algorithm is called Less-congested Open Shortest Path First (LOSPF). A simple feed-forward neural network is used to predict the congestion problem in the computer network links which are over utilized. The neural network is trained to predict congested links based on a given traffic pattern. These predictions are used to modify Dijkstr's algorithm to select the shortest paths, that excluding those links. Better resource utilization is obtained by applying the resulting paths. This is achieved throughout avoiding congested links and using other links that are underutilized. Comparison between performance of the suggested algorithm and the performance of OSPF that uses Dijkstra's algorithm to find the shortest path. Simulation results show that LOSPF performance is better in term of distributing links utilizations, and the queuing delay in the congested links. In spite of the superiority of the proposed LOSPF algorithm, its execution time is almost same as that of the OSPF 


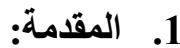

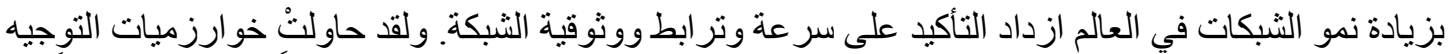

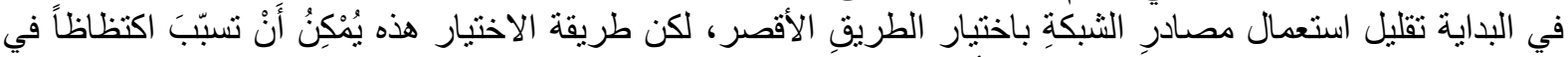

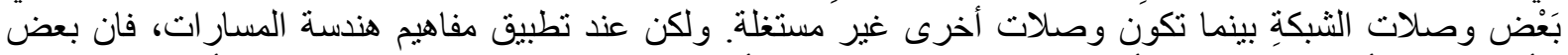

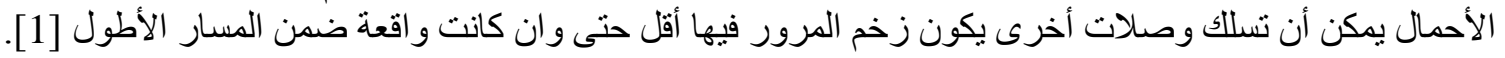

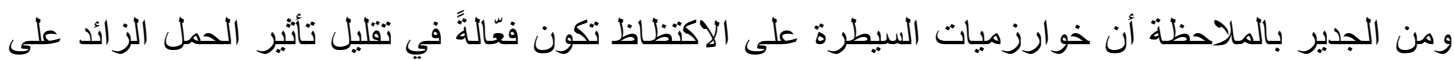

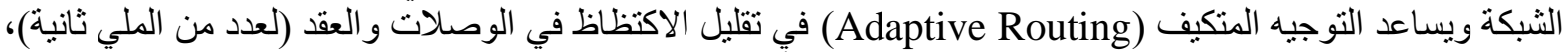

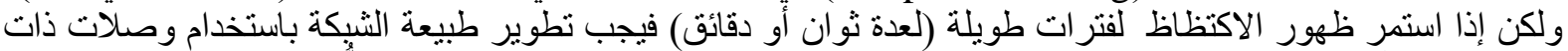

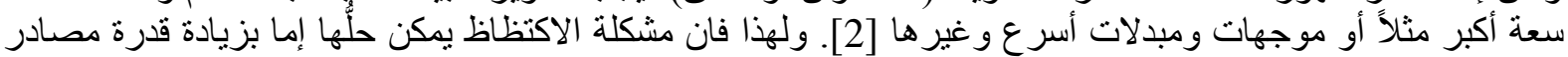

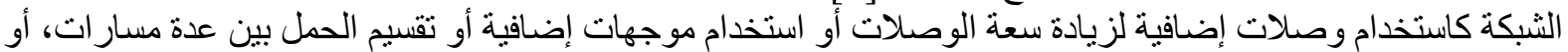

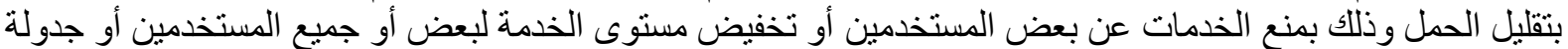

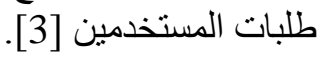

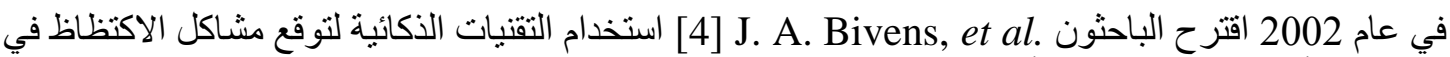

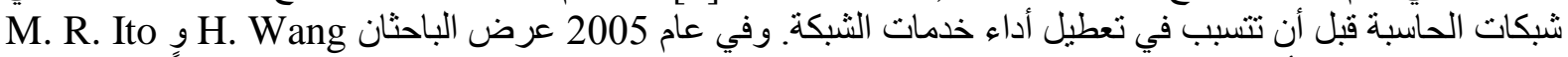

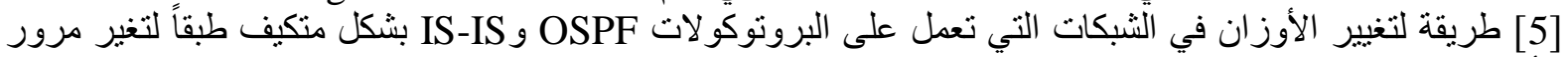

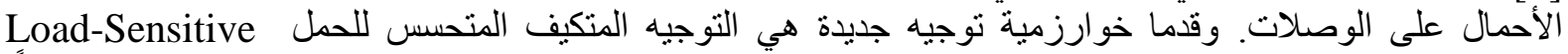

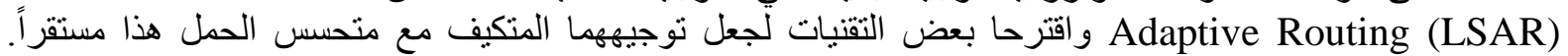

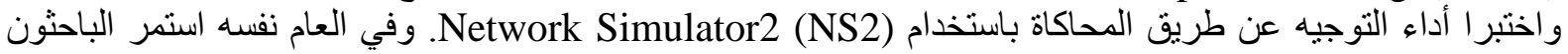

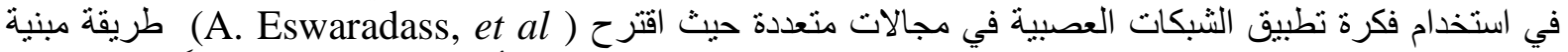

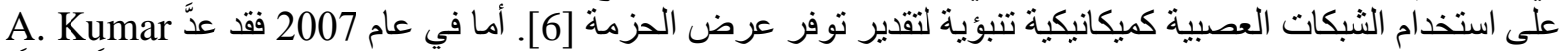

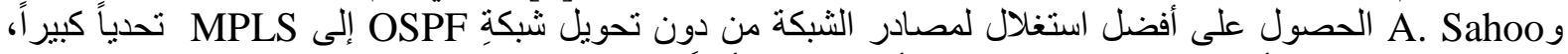
وقدما خو ارزمية ذكية أطلقا عليها خوارزمية "فتح أقصر مسار أو لاً الذكية" (Smart OSPF (S-OSPF) لتزود شبكة فيكة

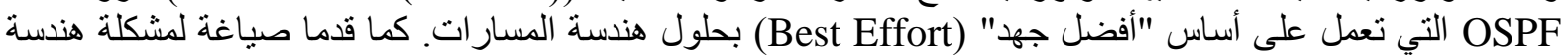

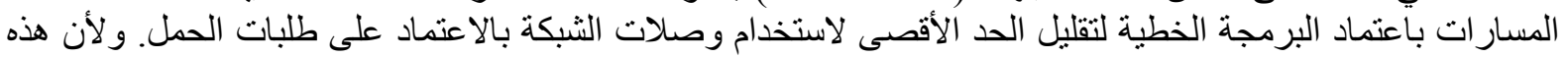

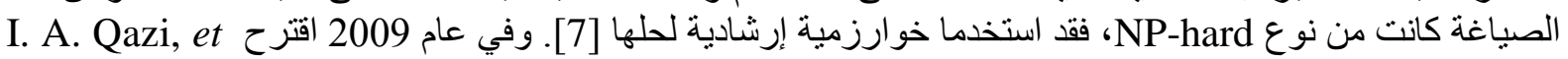
al هيكلية للسبطرة على الاكتظاظ في الثبكات سُميت "السيطرة على الني الاكتظاظ بالتوسيم الثنائي" ( Congestion Control

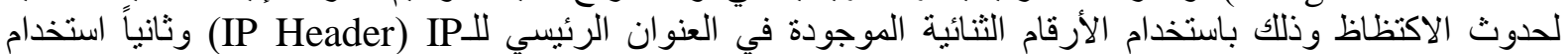

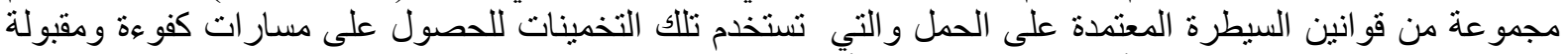

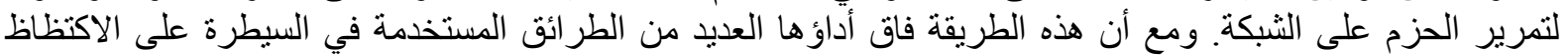

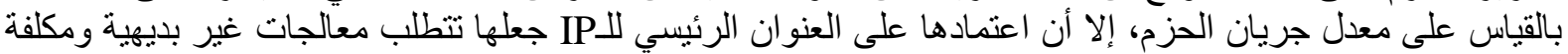

بالزمن [8].

في هذا البحث، بالإضافة الى مقطع المقدمة نم تعريف بعض بروتوكو لات التوجيه الداخلية في الثبكات السلكية

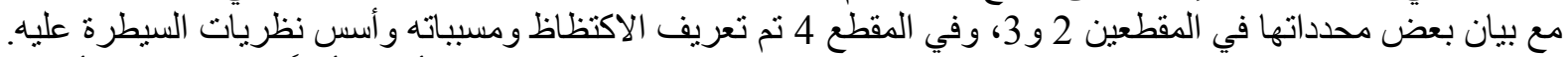

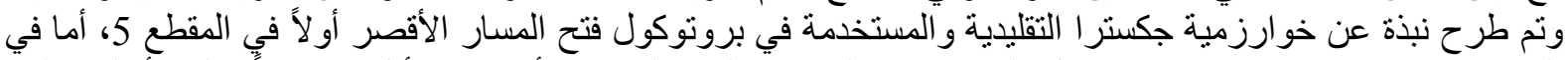

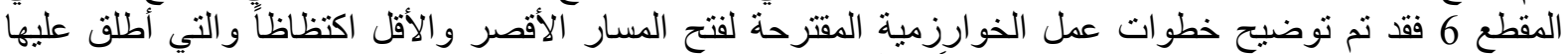

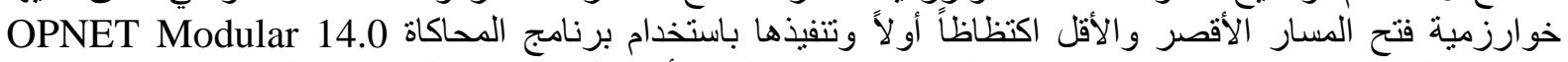

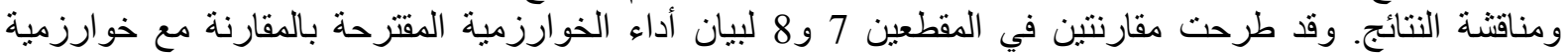

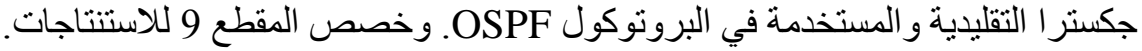

\section{2-بروتوكولات التوجيه الاخلية في الثبكات السلكية:}

\section{Routing Protocols In Intra-domain Wired Networks}

من أكثر بروتوكو لات التوجيه الداخلية (Intra-domain Wired Networks) استخداما في الوقت الحاضر

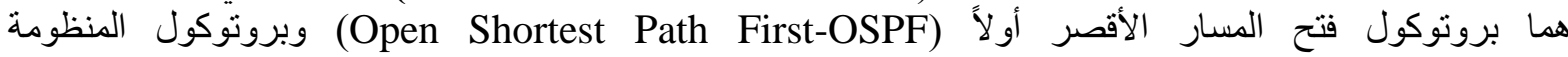

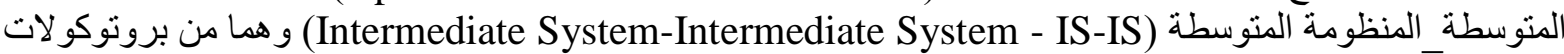


حالة الوصلة وكلاهما يوجه الرزم على طول المسار الأقصر المحسوب من نكاليف الوصلات (وتسمى أيضاً أوزان الوصلات) وبشكل مستقر تقريباً [9].

يتم تبادل المعلومات بين الموجهات من خلال الترويج لحالة الوصلة في الثبكة

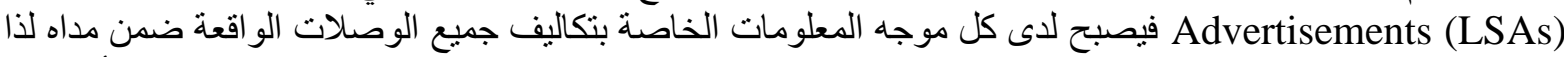

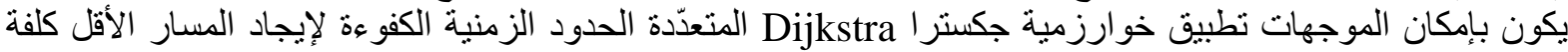

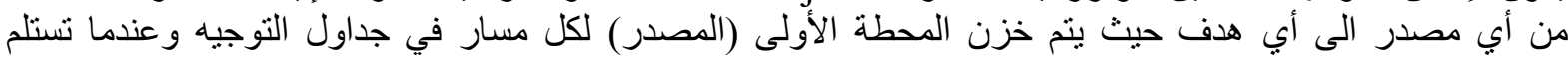

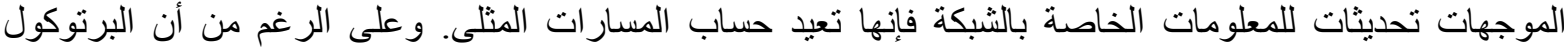
يوفران استخدام طريق واحد من الطرق الأقصر فإنه بإمكان كلا البرنوكولين استخدام

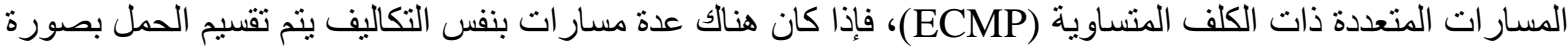

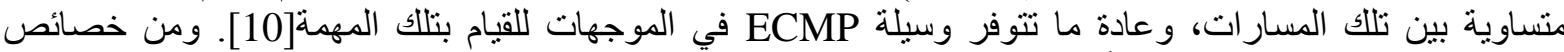

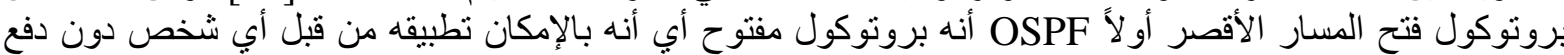

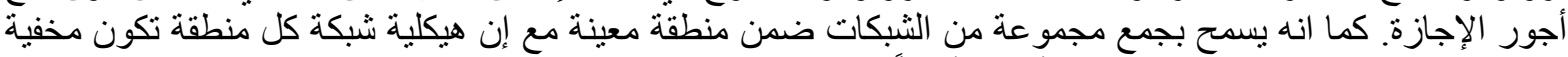

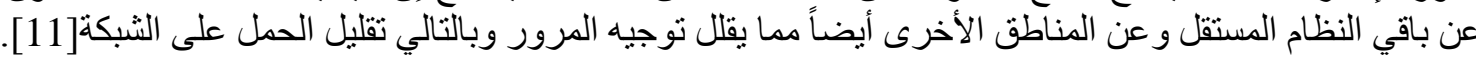
ولما كانت أوزان أو كلف الوصلات توضع من قبل مشغل الثبكة أو يتم حسابها باستخدام معادلة الكلفة الخاصة ببرونوكول فتح المسار الأقصر أو لاً التالية:

Metric $=\mathrm{K} 1 \times$ Bandwidth $+\mathrm{K} 3 \times$ Delay

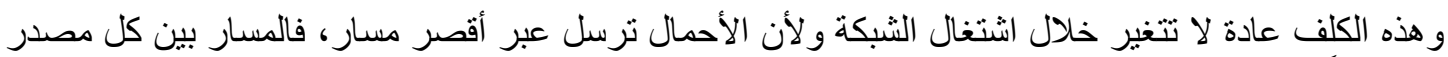

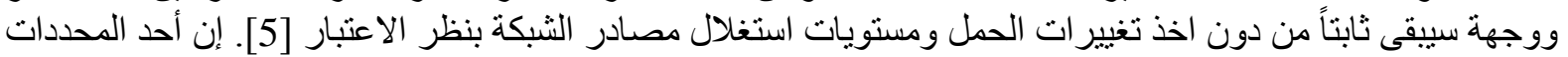

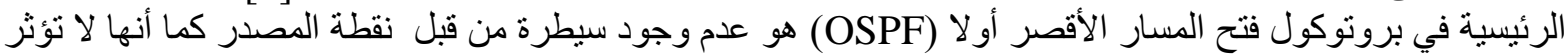

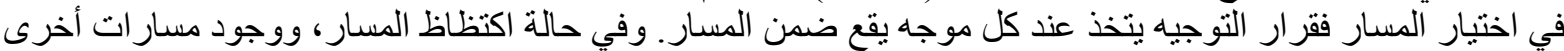
ولكنها ليست الأقصر ، فلا يتم استخدامها وان كانت غير مستغلة [12].

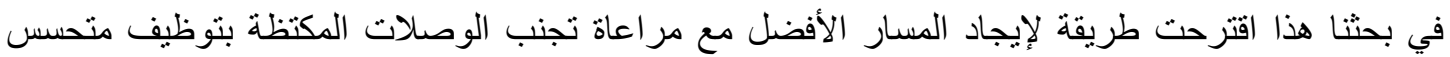
الأحمال باعتماد الثبكات العصبية في اكتشاف الاكتظاظ على الوصلات وذللك للتخلص من محددات بروتوكو لات التهات التوجيه

\section{2. محددات بروتوكولات التوجيه التقليدية:}

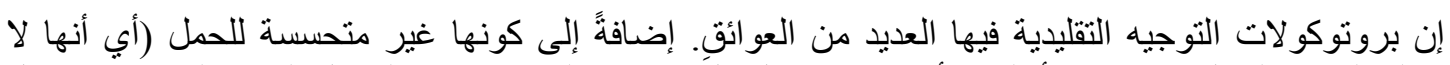

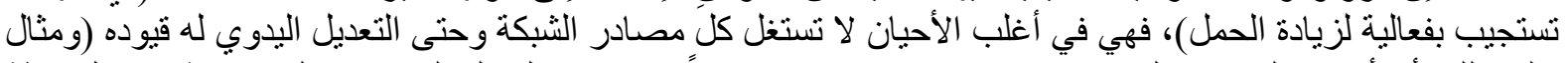

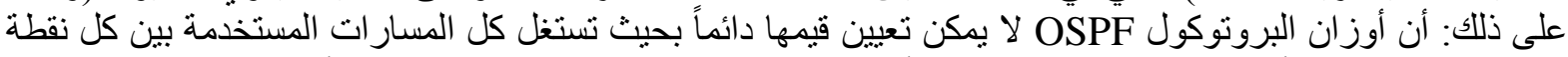

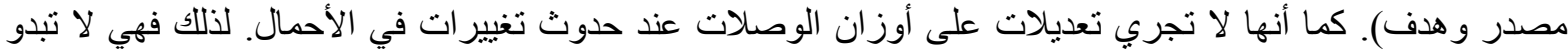

مرنة كفاية لدعم هندسة المسار ات [13]

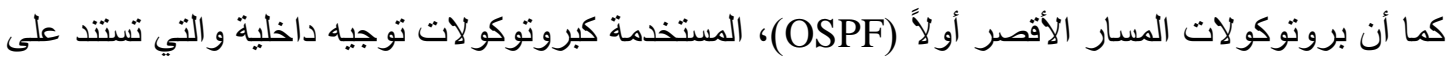
خوارزميات المسار الأقصر ، لها إمكانيات محدودة في مجال هندسة المسار اتل، وتتضمن هذه المحددات:

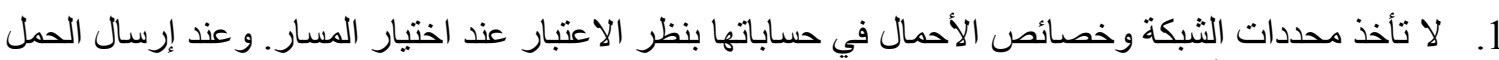
لا يمكنها تقسيم الأحمال بين مسار ات ذاتصن ذات كلف مختلفة.

2. قابلية المسار ات المتعددة للكلف المتساوية Equal-Cost Multi-Path (ECMP) وهي تدعم تقسيم الحمل بين

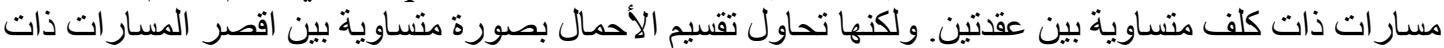

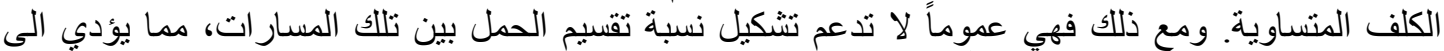

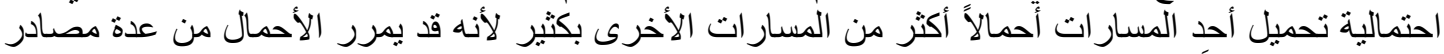

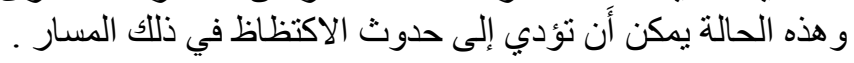

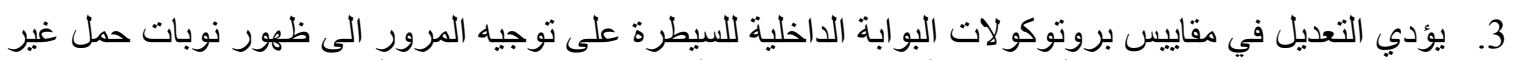

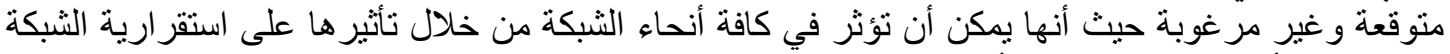

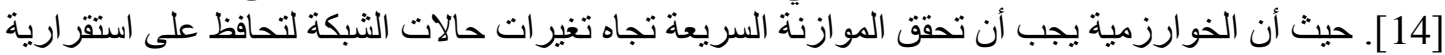

الثبكة [11] 
وقد عولجت مسألة ضعف مرونة برنوكول OSPF في هذه الدراسة ضمن الإطار الأصلي للأوزان في الوصلات

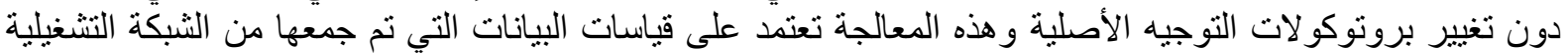

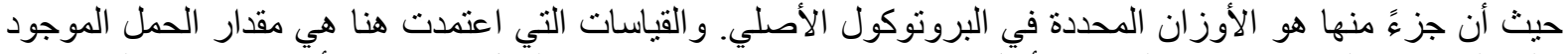

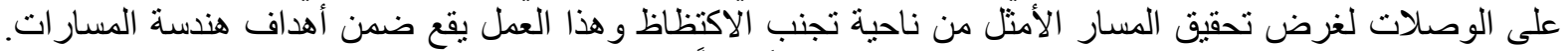
كما أن عدم تغيير أوزان الثبكة في هذه المعالجة سيكون عاملاً مهماً في الحفاظ على الألى استقر اريتها.

\section{4. الاكتظاظ وخوارزميات السيطرة عليه:}

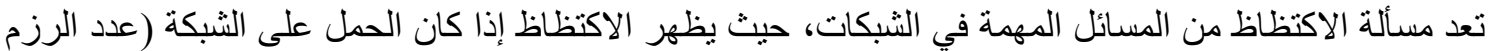

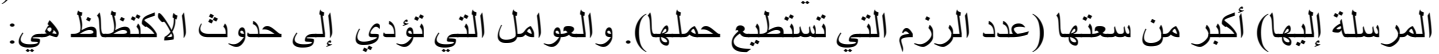

$$
\text { • معدل المرور الداخل يتعدى سعة الوصلات الخارجة. }
$$

• تكون الموجهات بطيئة جدا في تتفيذ مهاتها و التي تتمثل في تحديث الجداول و إدارة مستودعات الطو ابير.

$$
\text { • مستودعات الموجهات تكون محدودة جداً [2]. }
$$

أما مصطلح "السيطرة على الاكتظاظ" فيدل على الثقنيات المستخدمة لمر اقبة الثبكة و إدارة نقل بياناتها و إرسال

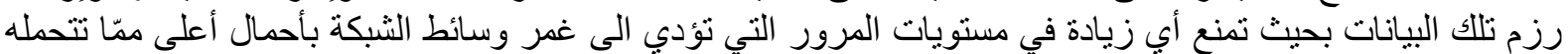

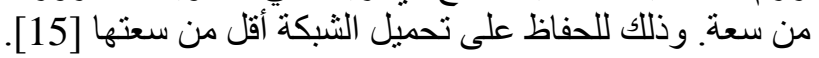

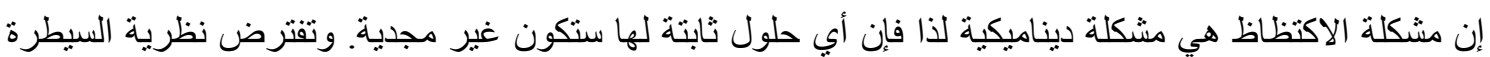

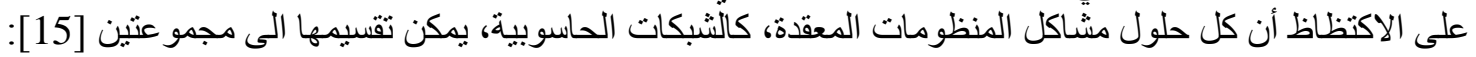

\section{• حلول الارات المفتوحة (Open Loop Solutions):}

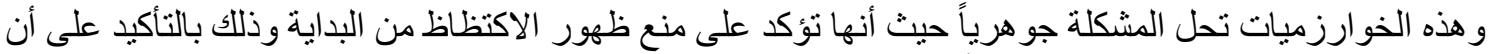

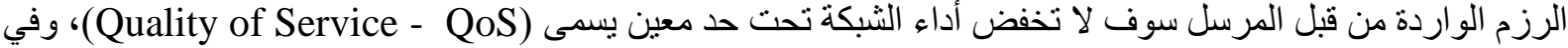

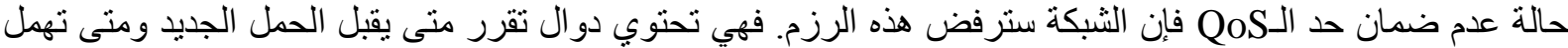

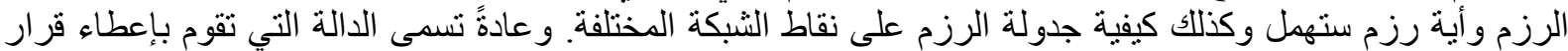

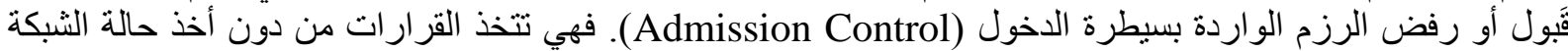

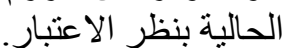

\section{• حلول الارات المغلقة (Closed Loop Solutions):}

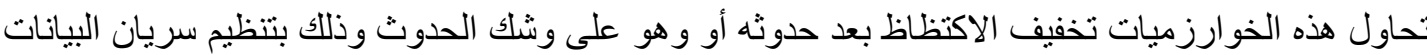

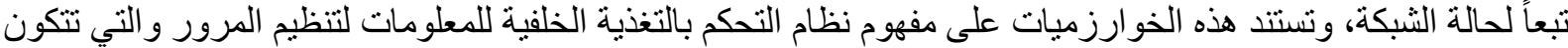

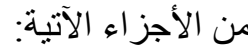

$$
\begin{aligned}
& \text { 1. مر اقبة النظام لكثف وقت ظهور الاكتظاظ وموقعه. } \\
& \text { 2. تمرير هذه المعلومات الى مو اقع اتخاذ القرارات. }
\end{aligned}
$$

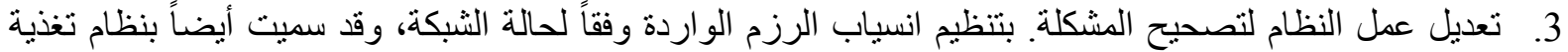
الداخل بالخارج لأن حالة الثبكة يجب أن تغذى ثانية الى النقطة أو مجمو عة النقاط التي تنظم المرور و عادة ما تكون

\section{5ijkstra's Algorithm خوارزمية جكسترا:}

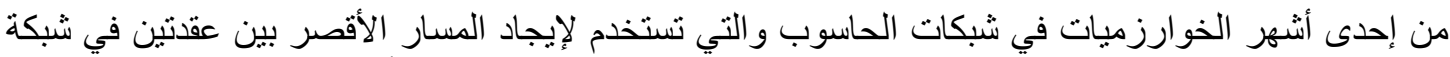

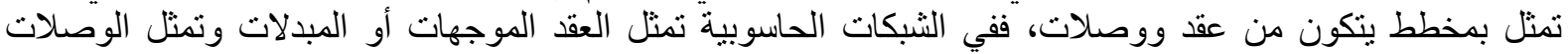
المسار ات وأوزان الوصلات تمثل الكلف [16].

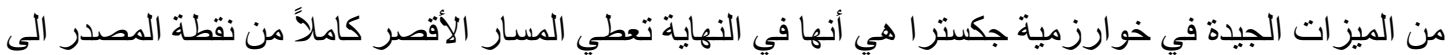

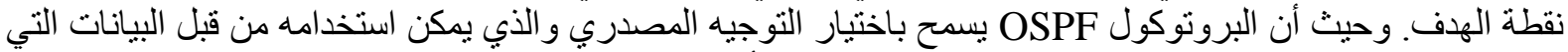
تستخدم المسار المحدد من قبل خوارزمية جكسترا فهو بالتأكيد يسمح باختيار الققزة القادمة في التوجيه المصدري التياني 


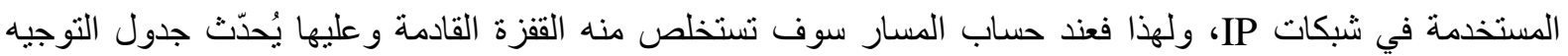
وبالتالي جدول الإرسال [17]. يمكن تمثيل خوارزمية جكستر ا في الموجهات كما موضح في فئه الثكل (1).

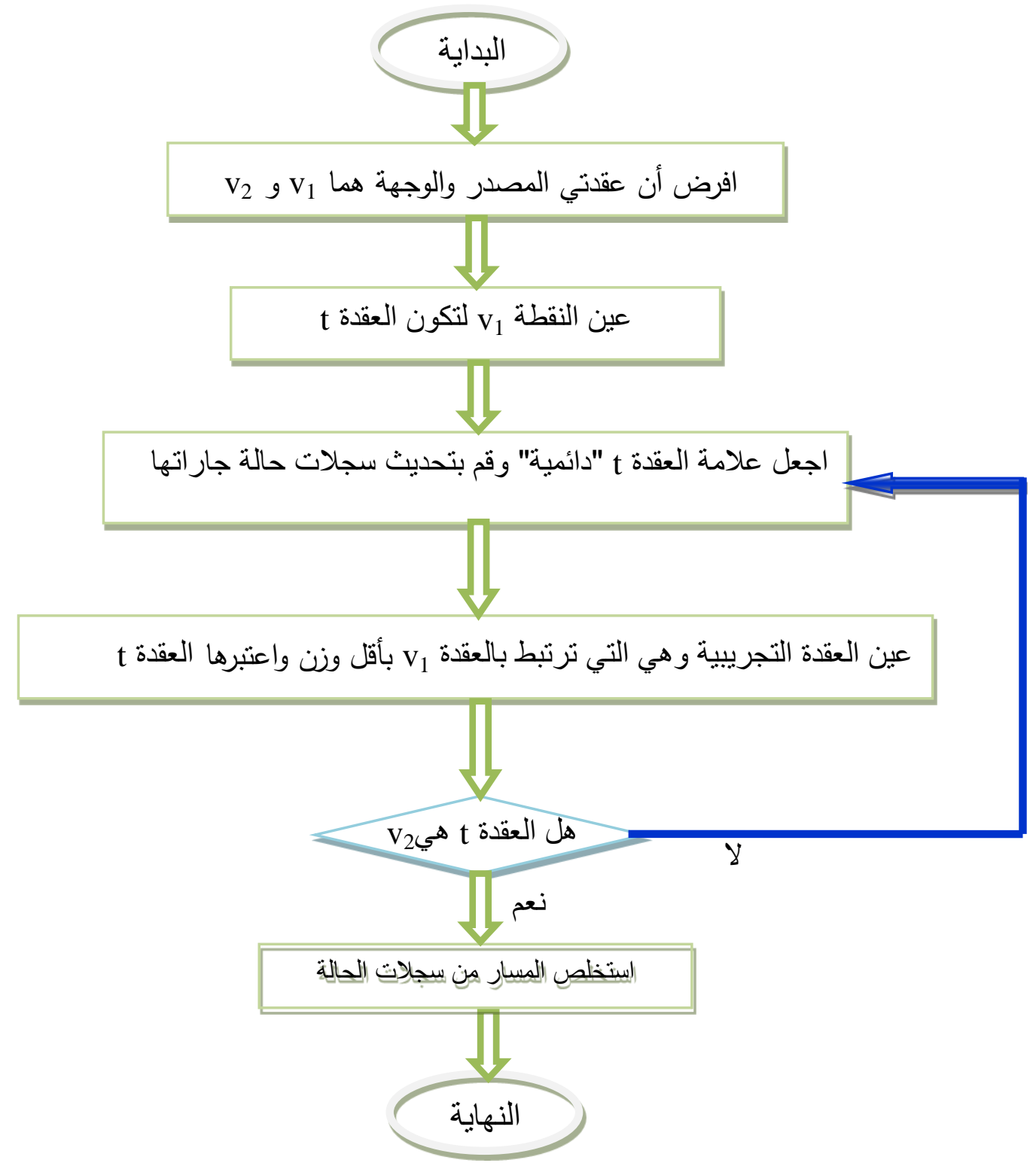

الثكل (1): المخطط الانسيابي لخوارزمية جكستر ا في الموجهات

6. خوارزمية فتح المسار الأقصر والأقل اكتظاظاً أولاً: Less-congested Open Shortest Path First (LOSPF)

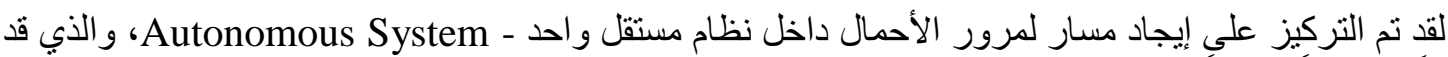

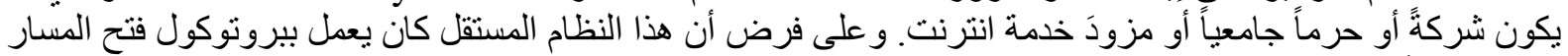

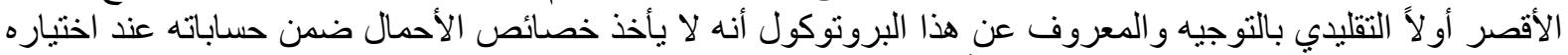

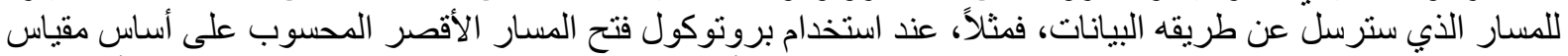

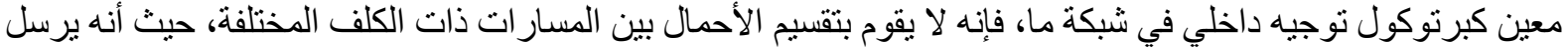


البيانات عبر المسار الأقصر دائماً دون مر اعاة اكتظاظه. ومع أن استخدام هذا البروتوكول يحافظ على مصادر الثبكة، و ولكنه يسبب المشاكل الآتية:

1. إذا تجاوز الحمل من نقطة مصدر الى نقطة هدف في الثبكة حدا معينا من استغلال أية وصلة و اقعة ضمن المسار

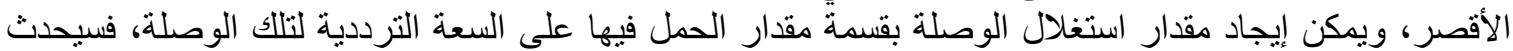

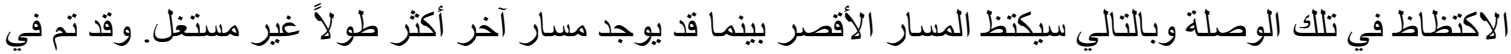
هذا البحث أعتماد 70\% من استغلال الوصلة كحد للاكتظاظ.

2. قد تتشارك المسارات الأقصر من نقاط مصادر مختلفة ببعض الوصداتلات، فإن تجاوز مجموع الأحمال لهذه النقاط السعة الترددية لتلك الوصلات فسوف يتسبب بحدوث الاكتظاظ.

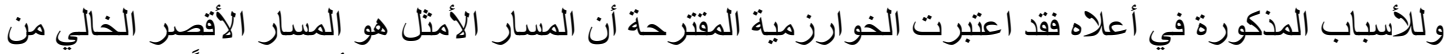

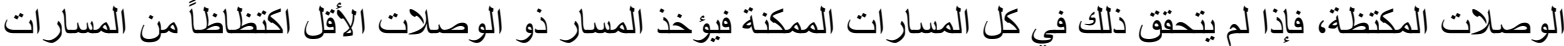

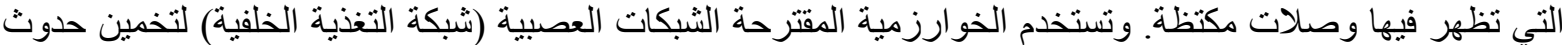

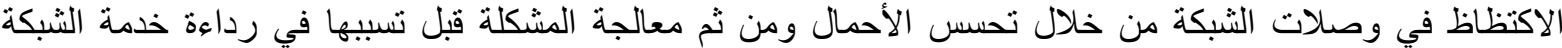

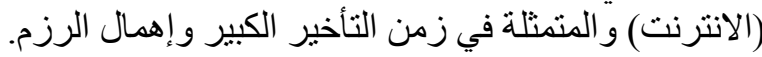

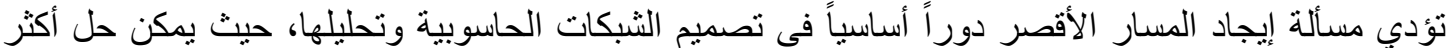

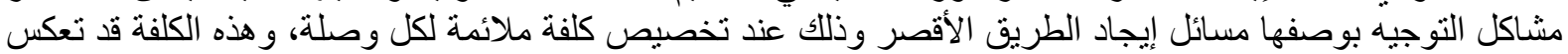

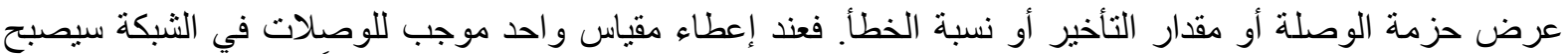

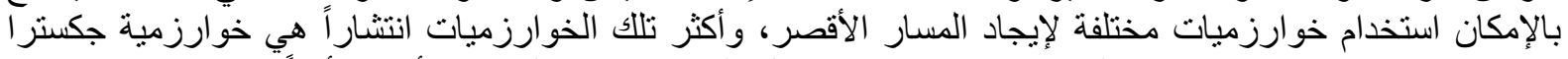

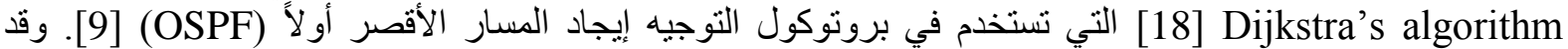

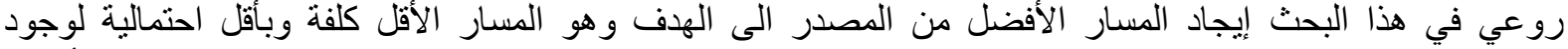

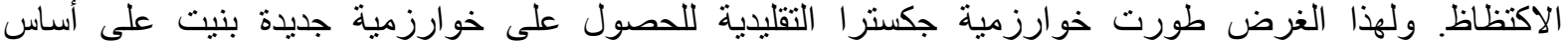

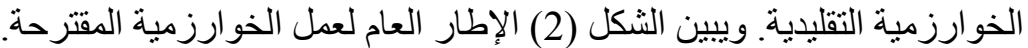

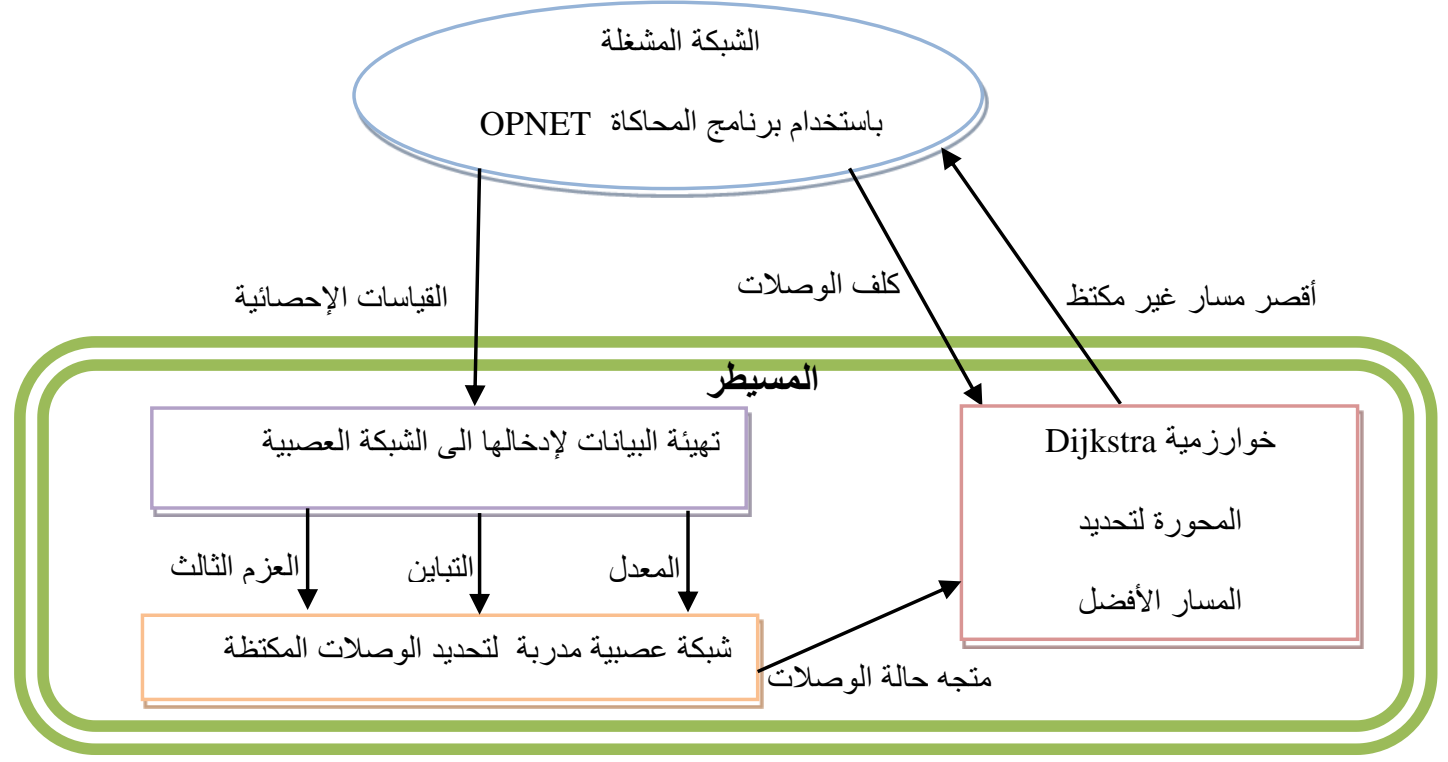

الثكل (2): الإطار العام لعمل الخوارزمية المقترحة

ففي مرحلة التهيئة تستخدم خوارزمية فتح المسار الأقصر و الأقل اكتظاظاً أو لاً (LOSPF) المقترحة نتيجة الثبكة

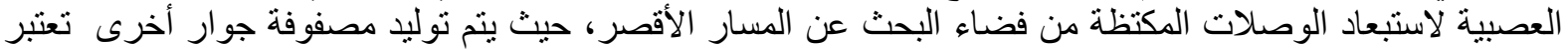

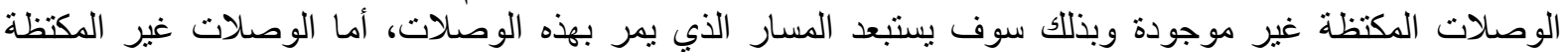

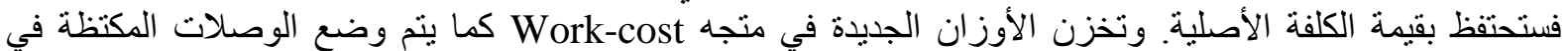

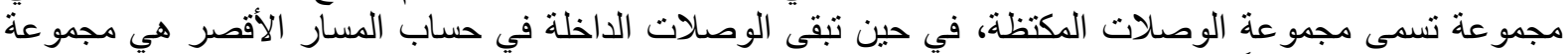
وصلات الثبكة مطروحاً منها مجمو عة الوصلات المكنة المكتظة. 
ثم في مرحلة المعالجة يتم استدعاء خوارزمية جكسترا التقليدية لإيجاد أقصر مسار ولكن بالاعنماد على مصفوفة الكية

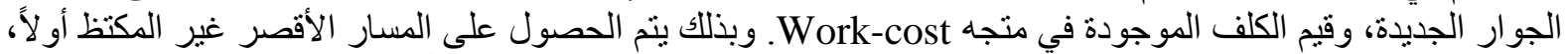

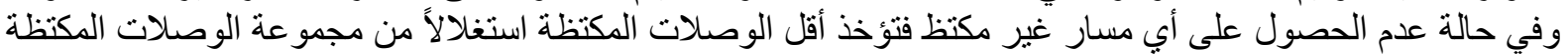

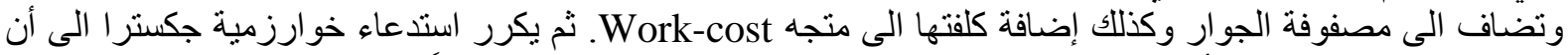

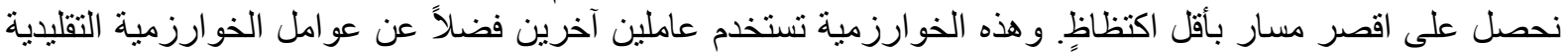

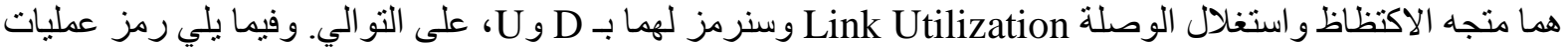

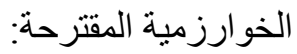

Less-congested Open Shortest Path First Algorithm Pseudo Code

LOSPF Algorithm ( G,w,D,U, s, t);

2

path = null;

CongestedLinks $=\{\}$;

For each link in $\mathrm{G}$

Work-cost $($ link $)=\mathrm{w}($ link $) / \mathrm{D}($ link $)$

If Work-cost (link) = infinity

Then CongestedLinks $=$ CongestedLinks + link

End

End

$10 \quad$ (path, cost $)=\operatorname{Dijkstra}(\mathrm{G}$, Work-cost, $\mathrm{s}, \mathrm{t})$

11 While (path = null) and (not empty(CongestedLinks)

$12 \quad$ Free-link = link from CongestedLinks with $\min (\mathrm{U})$

13 CongestedLinks $=$ CongestedLinks - Free-link

14 Work-cost(Free-link $)=\mathrm{w}($ Free-link $)$

$15 \quad$ (path, cost) $=\operatorname{Dijkstra}(\mathrm{G}$, Work-cost, $\mathrm{s}, \mathrm{t})$

16 End

17 Return (path, cost)

18 End

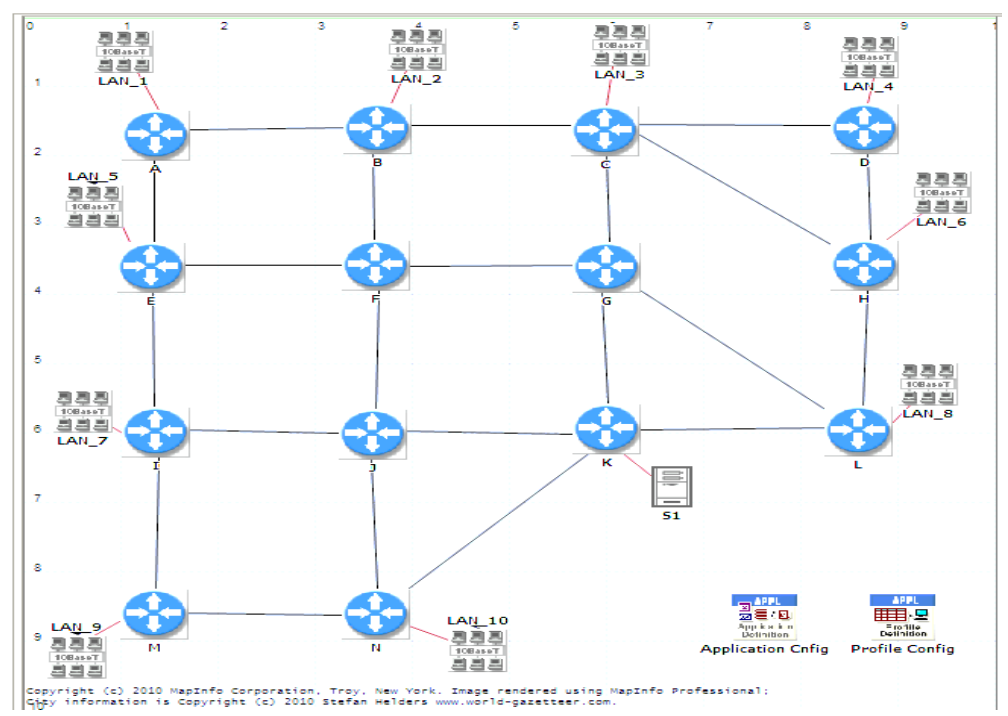

1-6 معطيات نموذج هيكلية الثبكة :

بالنسبة لهيكلية الثبكة الموضحة

في الثكل (3)، نم وضع نماذج طلبات مختلفة على الثبكة بشكل بحاكي زيادة أو قلة الطلبات خلال ساعات الدو ام الثختلفة، حيث أن لكل شبكة نماذج أحمال تتكرر تقريبا لتوريا

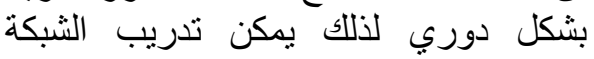

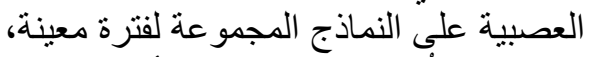

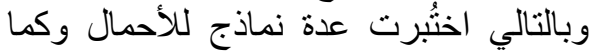
مبين في الجدول (1).

الشكل (3): نموذج هيكلية شبكة الحرم الجامعي المفترض 
الجدول (1): عدد طلبات الخدمة FTP الواردة في نموذج الثبكة موزعة حسب الساعات و عند كل LAN

\begin{tabular}{|c|c|c|c|c|c|c|c|c|c|c|c|}
\hline \multicolumn{12}{|c|}{ رموز الشبكات المحلية } \\
\hline \multirow{7}{*}{ 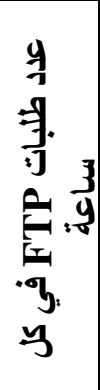 } & الساعات & $\begin{array}{l}\text { LA } \\
\text { N-1 }\end{array}$ & $\begin{array}{c}\text { LAN } \\
-2\end{array}$ & $\begin{array}{c}\text { LAN } \\
-3\end{array}$ & $\begin{array}{c}\text { LAN } \\
-4\end{array}$ & $\begin{array}{c}\text { LAN } \\
-5\end{array}$ & $\begin{array}{c}\text { LAN } \\
-6\end{array}$ & $\begin{array}{c}\text { LAN } \\
-7\end{array}$ & $\begin{array}{c}\text { LAN } \\
-8\end{array}$ & $\begin{array}{c}\text { LAN } \\
-9\end{array}$ & $\begin{array}{c}\text { LAN } \\
-10\end{array}$ \\
\hline & H1 & 20 & 75 & 50 & 20 & 25 & 75 & 10 & 15 & 25 & 10 \\
\hline & H2 & 40 & 50 & 60 & 40 & 50 & 75 & 25 & 15 & 35 & 20 \\
\hline & H3 & 50 & 25 & 75 & 50 & 70 & 25 & 50 & 25 & 50 & 25 \\
\hline & H4 & 50 & 25 & 75 & 50 & 70 & 25 & 40 & 25 & 45 & 25 \\
\hline & H5 & 25 & 50 & 40 & 25 & 50 & 25 & 40 & 15 & 35 & 10 \\
\hline & H6 & 10 & 75 & 40 & 10 & 25 & 50 & 25 & 15 & 25 & 5 \\
\hline
\end{tabular}

إن الأرقام في الجدول (1) تمثل عدد طلبات الخدمة الواردة في كل شبكة محلية وبالتالي فهي تمثل عدد الجلسات Sessions

و التي يجز ها الخادم المرتبط بالموجه Kن في هيكلية الثبكة كما في الثنكل (3).

\section{2-6 مدخلات الشبكة العصبية في الهيكلية ونتائجها:}

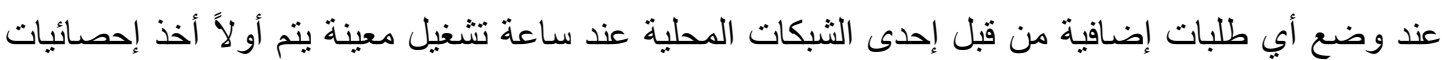

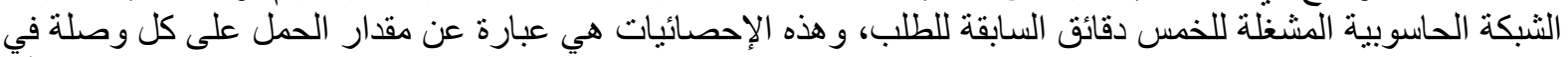

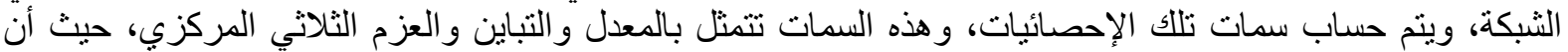

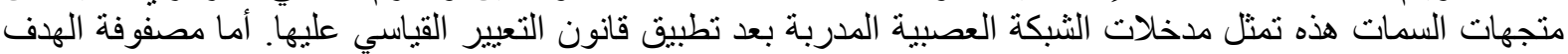
فتمثل أعلى حد لاستغلال الوصلة دون ان تعتبر مكتظة.

\section{3-6 استخدام نتائج الثبكة العصبية في إيجاد أقصر مسار غير مكتظ أولاً:}

لقد تم وضع طلبات خدمة إضافية على الثبكة وتوجيهها من الثبكات المحلية الى الخادم وذللك بنطبيق

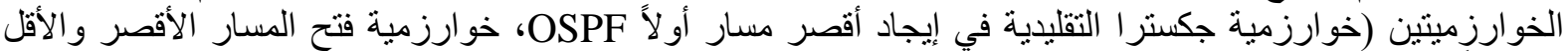

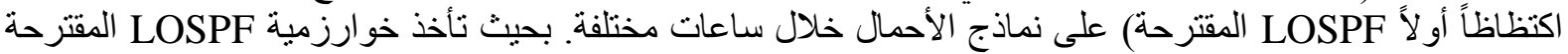

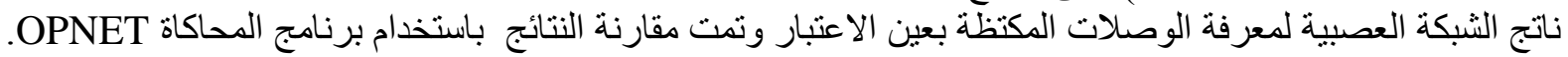

كما تمت مناقتنتها.

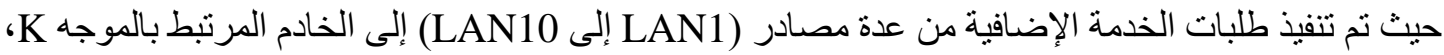

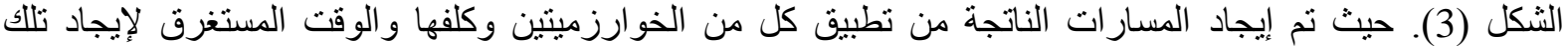

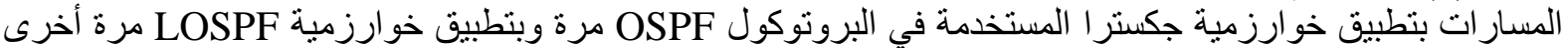

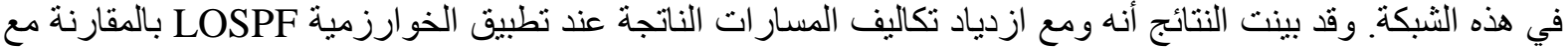

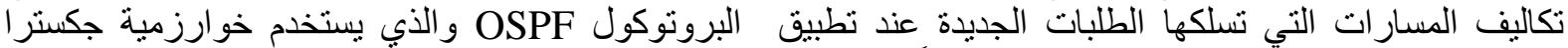

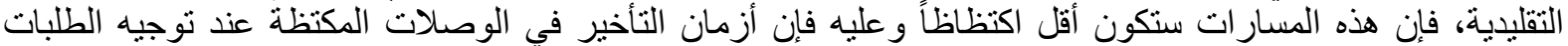

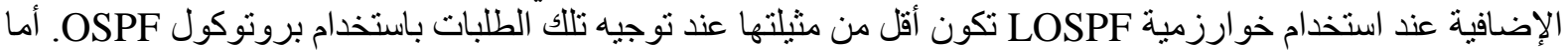

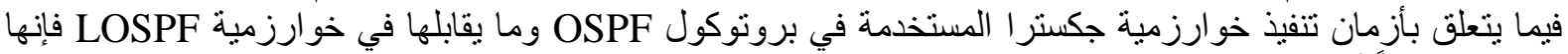

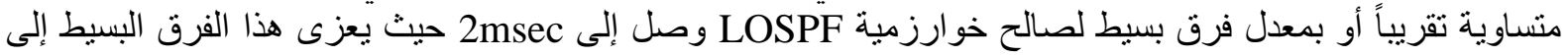
استبعاد الوصلات المكتظة من فضاء البحث في مصفوفة فر الجوار و والذي سيقلل من الوقت المستغرق للبحث.

\section{7. مقارنة استغلال الوصلات في نموذج هيكلية الثبكة:}

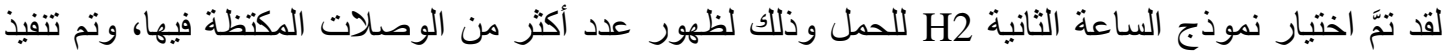

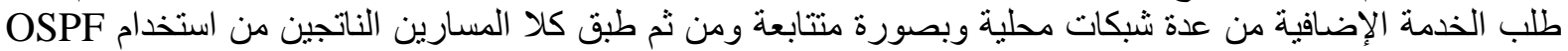

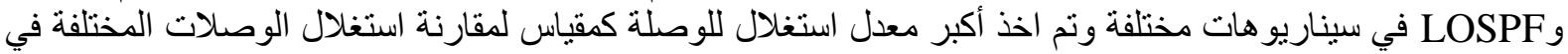

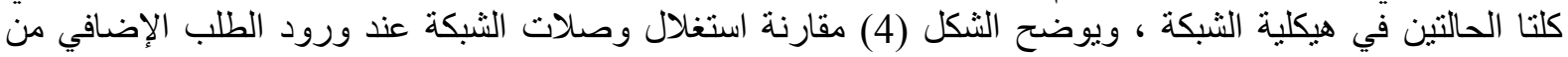


كشمولة: خوارزمية فتح المسار الأقصر والأقل اكتظاظاً أولاً في الثبكات الحاسوبية

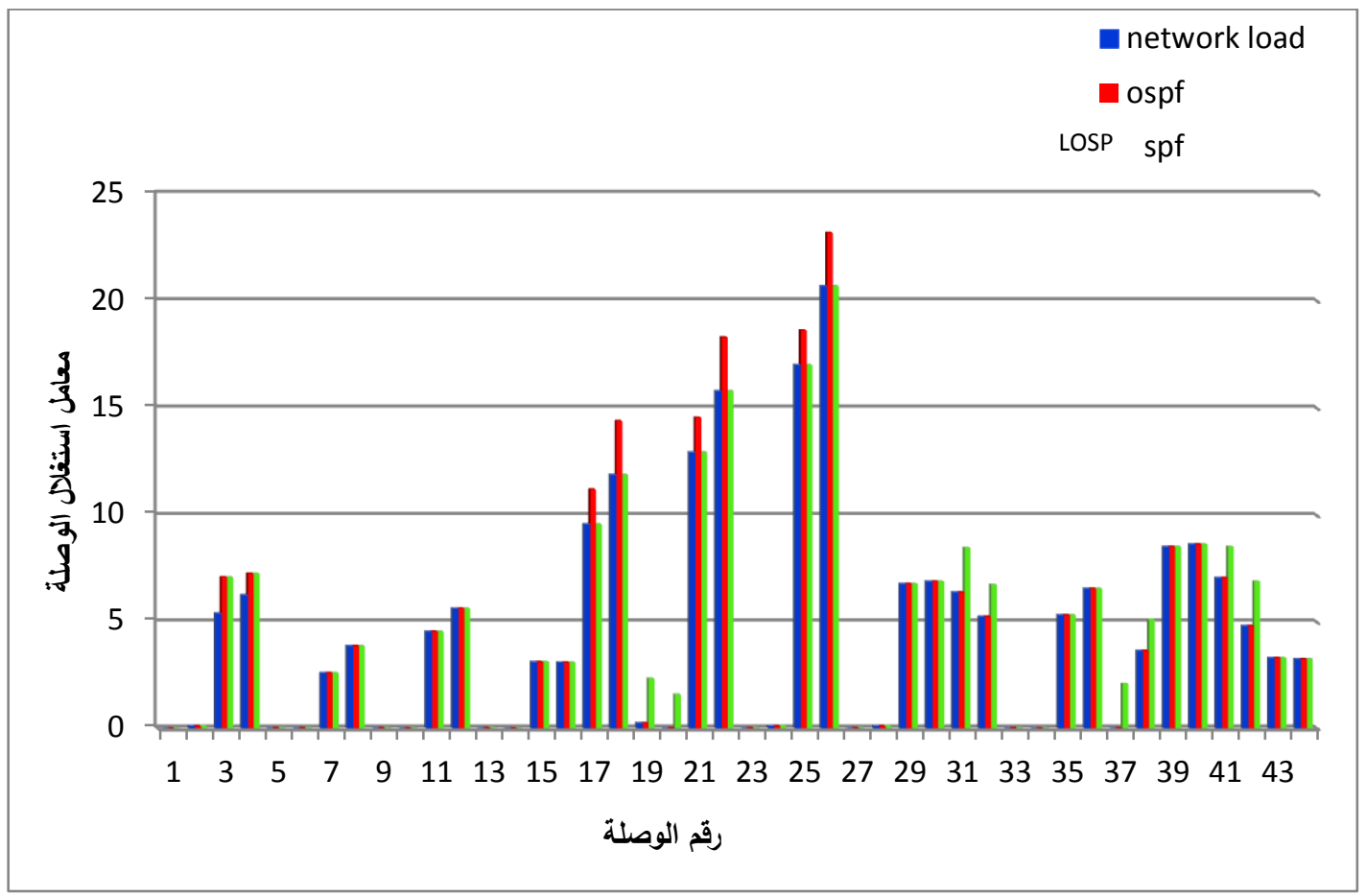

الثكل (4): مخطط مقارنة معامل استغلال الوصلات في الثبكة عند إضافة طلب من LAN1

8. مقارنة زمن التأخير في الوصلات المكتظة عند تطبيق المسارات الناتجة لكتا الخوارزميتين:

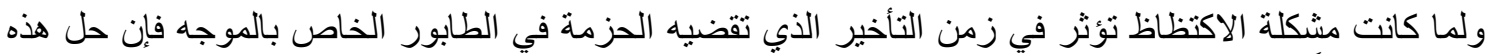

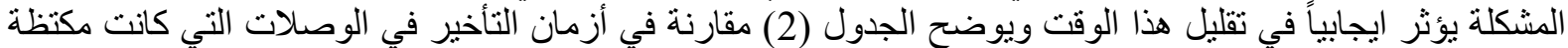

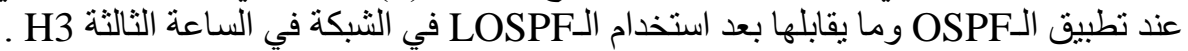

الجدول (2): أزمان التأخير في الوصلات التي

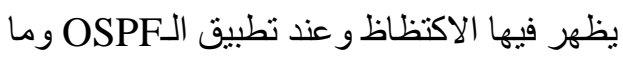

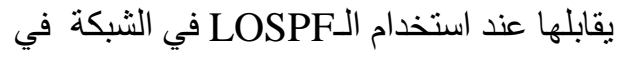
الساعة الثانية H2

\begin{tabular}{|c|c|c|c|}
\hline الوصد & الوصلة & في زمن التأخير & $\begin{array}{c}\text { زمن التأخير } \\
\text { LOSPF } \\
\text { (msec) }\end{array}$ \\
\hline 7 & $\mathrm{BF}$ & 12.573 & 6.675 \\
\hline 17 & $\mathrm{EF}$ & 7.292 & 5.563 \\
\hline 21 & FG & 5.033 & 3.981 \\
\hline 22 & GF & 6.97 & 4.355 \\
\hline 25 & GK & 7.64 & 5.335 \\
\hline 29 & HL & 9.076 & 6.476 \\
\hline 35 & $\mathrm{JK}$ & 7.477 & 4.572 \\
\hline 39 & KL & 19.252 & 12.764 \\
\hline 40 & LK & 8.716 & 5.962 \\
\hline
\end{tabular}

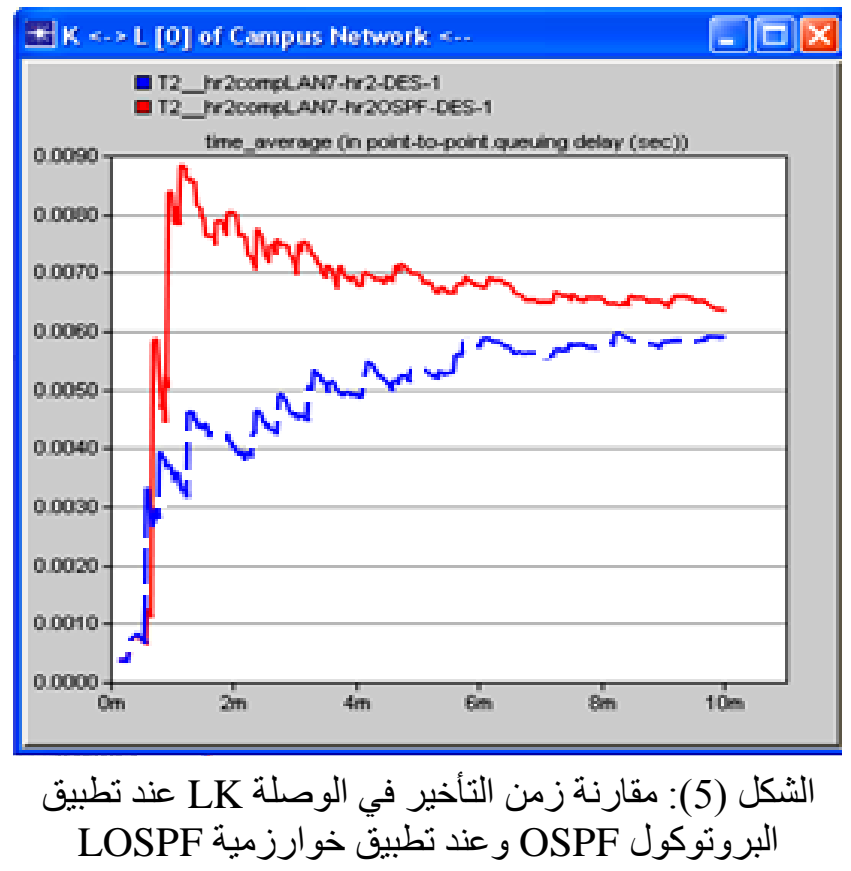


ويبين الثكل (5) قيم الفرق بين معدلات أزمان في إحدى الوصلات المكنظة.

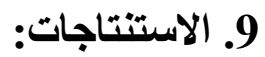

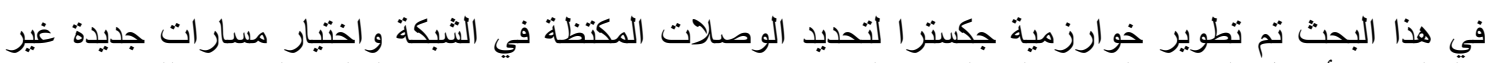
مكتظة لطلبات الأحمال الجديدة الواردة الى الثبكة الحاسوبية. و استخدمت شبكة الانتشار الخلفي العصبية للتنتبؤ بحدوث

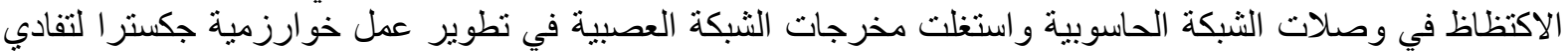

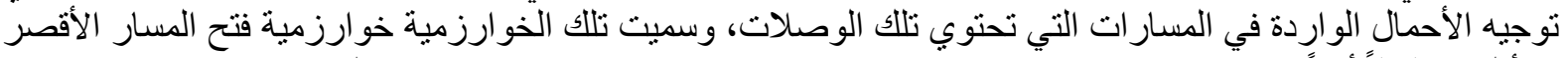
و الأقل اكتظاظاً أو لاً (Less-congested Shortest Path Firs LOSPF). ونم استنتاج مايلي :

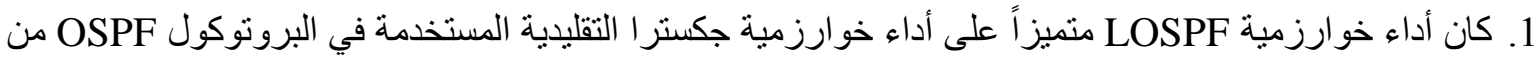

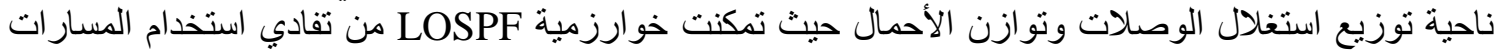

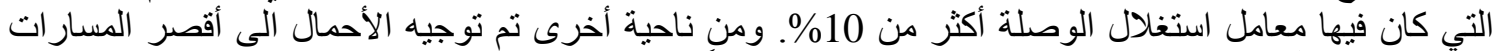

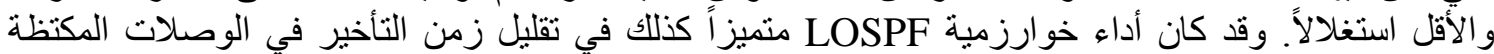

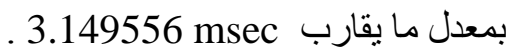

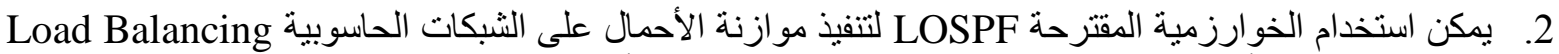

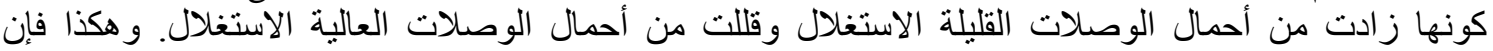

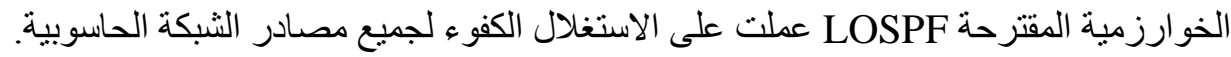

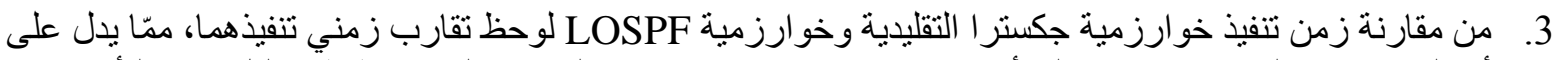

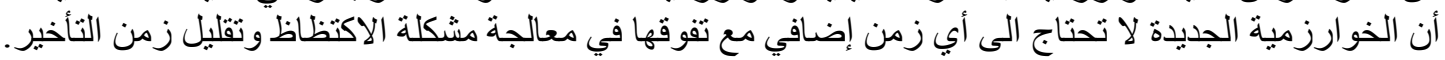
4. عند تطبيق الخوارزمية الجديدة بنماذج أحمال مختلفة لوحظ تمكنها من الأداء الجيد مما يدل على على إمكانية استخدام تلأك الخو ارزمية في شبكات الأنظمة المستقلة.

1. Piedra N., Chicaiza J., López J. and García J., (2008), "Study of The Application of Neural Networks In Internet Traffic Engineering", Sixth International Conference on Information Research and Applications- i. Tech, Varna, Bulgaria.

2. Sharma R.,(2009), "Computer Network", University Science Press An Imprint of Laxmi Publications Pvt. Ltd. 113, Golden House, Daryagani, New Delhi-110002.

3. Rekhter Y. and Li T., (1995), " A Border Gateway Protocol 4 (BGP-4)", Network Working Group Request for Comments: 1771.

4. Alan bivens J., Szymanski B. K. and Embrechts M. J., (2002), "Network Congestion Arbitration and Source Problem Prediction Using Neural Networks", Smart Engineering System Design. Vol. 4; pp. 243-252.

5. Wang H. and Ito M. R., (2005), "Dynamics of Load Sensitive Adaptive Routing", Proceedings of the IEEE International Conference on Communications (ICC), Vol. 1, pp. $213-217$.

6. Eswaradass A., Sun X. and Wu M., (2005), "A Neural Network Based Predictive Mechanism for Available Bandwidth". IEEE, The ACM Digital Library. http://www.cs.iit.edu/ scs/psfiles/AlakSunWuIPDPS2005.pdf

7. Mishra A. K. and Sahoo A., (2007), 'S-OSPF: "A Traffic Engineering Solution for OSPF Based Best Effort Networks", IEEE International Conference on Global Communications (Globecom), Washington http://www.cse.iitb.ac.in/ sahoo/papers/globecom2007_aditya.pdf

8. Qazi I. A. , Znati T. and Andrew L. L. H., (2009), "Congestion Control Using Efficient Explicit Feedback", proceedings of the IEEE Communication Society subject matter 
experts for publication in the IEEE INFOCOM, Rio de Janeiro, Brazil. http://leecenter.caltech.edu/workshop09/papers/low7.pdf

9. Moy J., (1998), "OSPF Version 2", Network Working Group Request for Comments, RFC 3630. http://www.faqs.org/rfcs/rfc2328.html

10. Skrypnyuk N., (2006), " Load-Sensitive Routing", Diplomarbeit in Informatik, Technische Universität München, Garching bei München, Germany, http://www.net.tlabs.tu-berlin.de/papers/S-LSR-06.pdf

11. Macfarlane J., (2006), " Network Routing Basics: Understanding IP Routing in Cisco® Systems", Published by Wiley Publishing, Inc., Indianapolis, Indiana.

12. Ronaldo M. and Vitor G., (2007), "Efficient Routing Heuristics for Internet Traffic Engineering", Elsevier B.V., Computer Communications 30, pp. 1942-1952.

13. Fortz B., Rexford J. and Thorup M., (2002), "Traffic Engineering With Traditional IP Routing Protocols", IEEE Communication Magazine, Vol. 40, No. 10, pp.118-124. http://www.cs.princeton.edu/ jrex/papers/ieeecomm02.pdf

14. Awduche D., Chiu A., Elwalid A., Widjaja I. and Xiao X., (2002), "Overview and Principles of Internet Traffic Engineering", Network Working Group Request for Comments: RFC 3272. http://www.tools.ietf.org/html/rfc3272.

15. Kalunga J., (2006), "What is Congestion Control in Broadband Networks?", Connexions module: m13361, Version 1.2, US / Central. http://cnx.org/content/m13361/1.2/.

16.Sniedovich M., (2006), "Dijkstra's Algorithm Revisited: The Dynamic Programming Connexion, Department of Mathematics and Statistics", vol. 35 No. 3, Department of Mathematics and Statistics, The University of Melbourne, Australia.

17. Medhi D. and Ramasamy K., (2007), "Network Routing Algorithms, Protocols, and Architectures ", Morgan Kaufmann Publishers, imprint of Elsevier.500 Sansome Street, Suite 400, San Francisco.

18. Dijkstra E, (1959), "A Note on Two Problems in Connexion with Graphs", NumerischeMathematik, Vol. 1, pp. 269-271.

تم اجزاء الُبحث في كلية الهُذسة = جامعة الموصنل 